\title{
Deep near-infrared photometry of the globular cluster 47 Tucanae. Reconciling theory and observations ${ }^{\star}$
}

\author{
M. Salaris ${ }^{1}$, E. V. Held ${ }^{2}$, S. Ortolani ${ }^{3}$, M. Gullieuszik ${ }^{2}$, and Y. Momany ${ }^{2}$ \\ 1 Astrophysics Research Institute, Liverpool John Moores University, Twelve Quays House, \\ Egerton Wharf Birkenhead CH41, 1LD, UK \\ e-mail: ms@astro.livjm.ac.uk \\ 2 Osservatorio Astronomico di Padova, INAF, vicolo dell'Osservatorio 5, 35122 Padova, Italy \\ e-mail: [enrico.held;marco.gullieuszik; yazan.almomany]@oapd.inaf.it \\ 3 Dipartimento di Astronomia, Università di Padova, vicolo dell'Osservatorio 3, 35122 Padova, Italy \\ e-mail: sergio.ortolani@unipd.it
}

Received 8 August 2007 / Accepted 3 October 2007

ABSTRACT

\begin{abstract}
Context. The Galactic globular cluster 47 Tucanae is central to studies of Galaxy formation, and a test-bed for theoretical models, distance determination and extragalactic age-dating techniques. Independent parallax-based distance determinations in the optical spectral range provide discrepant results; also, star counts along the Red Giant Branch from optical data have disclosed a worrying disagreement with theoretical predictions, that impacts not only the theory of red giant stars, but also the calibration of the age scale of extragalactic systems.

Aims. Our new near-infrared data for 47 Tuc set constraints on its distance and test the reliability of theoretical red giant branch star counts, independently of previous conclusions from optical work.

Methods. We have obtained deep near-infrared imaging of 47 Tuc using SOFI at the ESO New Technology Telescope. Colour-magnitude diagrams, isochrones and synthetic horizontal branch modelling have been used to determine the distance of 47 Tuc and constrain its age. We have also constructed a luminosity function of red giant stars, which has been compared with theoretical predictions of stellar evolution models.

Results. We obtain a distance $(m-M)_{0}=13.18 \pm 0.03($ random $) \pm 0.04$ (systematic), for $[\mathrm{Fe} / \mathrm{H}]=-0.7 \pm 0.1$ and $E(B-V)=$ $0.04 \pm 0.02$. This supports the shorter end of the range of distances obtained from optical studies. The mean horizontal branch star mass is between 0.65 and $0.66 M_{\odot}$, and its $1 \sigma$ Gaussian dispersion is between 0.010 and $0.012 M_{\odot}$. The cluster age can only be approximately estimated from the data, and is between $\sim 10$ and $\sim 13 \mathrm{Gyr}$. The luminosity function of red giant branch (and early-asymptotic giant branch) stars does not show a statistically significant discrepancy with theory. The brightness of the red giant branch bump in the near-infrared is possibly fainter than the models, although the uncertainty on the spectroscopic metallicity and age prevents to reach a firm conclusion on this issue.
\end{abstract}

Key words. globular clusters: individual: 47 Tuc - infrared: stars - stars: distances - stars: luminosity function, mass function stars: population II

\section{Introduction}

The metal-rich globular cluster 47 Tucanae (NGC 104) has played and continues to play a fundamental role in both Galactic and extragalactic studies. First of all, 47 Tuc belongs to the "thick disk" population of Galactic globular clusters (GCs), and the comparison of its age with that of the more metal-poor "halo" GCs and the oldest "thin disk" open clusters provides clues about the timescale for the formation of the Galactic stellar populations (Salaris \& Weiss 1998; Liu \& Chaboyer 2000; VandenBerg 2000; Salaris et al. 2004b). It also provides the zero point for the age determination of "bulge" GCs, since their ages are most reliably determined from the differential comparison of their colour-magnitude diagrams (CMDs) with that of 47 Tuc (Ortolani et al. 1995). Moreover, 47 Tuc has been used as testbed to compare different distance determination methods (such as white dwarf-fitting, main sequence-fitting, red clump method; see, e.g., Zoccali et al. 2001; Percival et al. 2002, and references

$\star$ Based on data collected at the European Southern Observatory, La Silla, Chile, Proposal 66.B-0247. therein) employed to calibrate the Galactic and extragalactic distance scale.

The integrated properties of 47 Tuc play an important role for testing methods to estimate the mean age and metallicity of extragalactic systems. To derive reliable information about stellar age and metallicity from the integrated light of unresolved galaxies one must overcome the age-metallicity degeneracy, which affects both integrated colours and absorptionline strengths (Worthey 1994). Age-dating techniques based on the Balmer lines (Jones \& Worthey 1995) have shown great promise in breaking this degeneracy, but they need to be tested on Galactic GCs for which independent age estimates based on their resolved stellar populations are possible. Gibson et al. (1999) applied the $\mathrm{H}_{\gamma}-\mathrm{Fe}_{4668}$ line indices diagram to the determination of the age of 47 Tuc from its integrated spectrum, obtaining an age well in excess of $20 \mathrm{Gyr}$, much larger than CMD ages, currently estimated in the range 11-13 Gyr.

Vazdekis et al. (2001) and Schiavon et al. (2002) have investigated this issue in detail. In their analysis of the problem, Schiavon et al. (2002) compared the observed differential 
luminosity function (star counts as a function of magnitude - LF) of the cluster's stars, with theoretical counterparts from different authors; this comparison disclosed a worrying discrepancy along the upper red giant branch (RGB) that, according to the authors, could be one of the main causes of the spectroscopicCMD age problem. The predicted number of RGB stars above the horizontal branch appears to be about a factor of 2 lower than observed. This discrepancy found by Schiavon et al. (2002) adds to the discrepancy between predicted and observed values of the quantity $R_{\text {bump }}$ (ratio between star counts across the RGB bump and fainter RGB stars) introduced by Bono et al. (2001). The observed value of $R_{\text {bump }}$ for 47 Tuc is more than $2 \sigma$ larger than the theoretical predictions, whereas for almost all other clusters in the sample of Bono et al. (2001) no significant disagreement is found. Given that the luminosity function of RGB stars tests the composition stratification above the outward moving thin H-shell (Renzini \& Fusi Pecci 1988; Cassisi et al. 2002), differences between predicted and observed RGB luminosity functions may be caused by additional physics (e.g. rotation and/or additional element transport mechanisms) not included in the model computations.

In this paper we present the deepest to date near-infrared (near-IR) CMD of $47 \mathrm{Tuc}$, in the 2MASS $J H K_{\mathrm{s}}$ system; its well populated RGB allows us to reassess the extent of the disagreement between observed and predicted star counts along the cluster RGB. Apart from minimising the effect of extinction, the advantage of using near-IR filters is that they bracket the spectral region of maximum flux density for RGB stars, plus the bolometric corrections are essentially unaffected by the star chemical composition. We also provide a new estimate of the cluster distance and mean mass loss along the RGB, by fitting synthetic horizontal branch (HB) models to the observed counterpart. Constraints on the cluster age from the Turn Off region of the CMD will also be discussed. The whole theoretical analysis has been performed employing the recent and widely used BaSTI library of stellar models and isochrones by Pietrinferni et al. (2004, 2006).

The paper is structured as follows. Section 2 describes the observations and data reduction procedures, while the observed CMD and luminosity function are presented in Sect. 3. Section 4 compares the CMD with theoretical isochrones and presents a determination of the cluster distance and age. The comparison of observed and predicted star counts is performed in Sect. 5, followed in Sect. 6 by a summary of the results and conclusions.

\section{Observations and data reduction}

\subsection{Observations}

Near infrared $J H K_{\mathrm{s}}$ observations of 47 Tuc were carried out on Nov. 12, 2000 under stable photometric conditions and seeing $<0.9$ arcsec. We used the SOFI infrared camera mounted at the ESO/NTT, equipped with a Hawaii HgCdTe $1024 \times 1024$ pixels array detector. The large field mode with 0.29 pixel was used for all observations, yielding a 4'.9 $\times 44^{\prime} .9$ field-of-view. The readout mode was Double Correlated Read, with a readout noise 2.1 ADU and gain $5.53 \mathrm{e}^{-} / \mathrm{ADU}$.

Table 1 reports the journal of observations of 47 Tuc. We obtained deep and shallow imaging to increase the dynamic range of our observations, in order to measure bright red giant branch (RGB) stars as well as faint stars below the main sequence (MS) turnoff (TO). However, despite using for the shallow images the shortest detector integration time (DIT) permitted by the instrument, the brightest RGB stars were saturated. The deep
Table 1. The journal of observations of 47 Tuc.

\begin{tabular}{lccccc}
\hline \hline Field & RA(J2000) & Dec(J2000) & Filter & $N_{\text {ima }}$ & DIT $\times$ NDIT (s) \\
\hline deep & $00: 24: 09.5$ & $-72: 02: 59$ & $J$ & 3 & $10 \times 6$ \\
& & & $H$ & 3 & $5 \times 12$ \\
& & $K_{\mathrm{s}}$ & 3 & $5 \times 12$ \\
shallow $00: 24: 08.7$ & $-72: 03: 53$ & $J$ & 3 & $1.18 \times 10$ \\
& & & $H$ & 3 & $1.18 \times 10$ \\
& & & $K_{\mathrm{s}}$ & 3 & $1.18 \times 10$ \\
\hline
\end{tabular}

exposures were obtained about $2^{\prime}$ off the cluster centre to avoid the most crowded regions, while the offset was somewhat smaller for the shallow exposures. A typical observing sequence in each filter consists of 3 images of 47 Tuc interspersed with 3 frames on offset sky positions. The center of our 47 Tuc pointings is given in Table 1, together with the number of images in each filter (3 dithered images were obtained in each of the $J H K_{\mathrm{s}}$ filters), and on-target total exposure times given as the product of DIT $\times$ NDIT (the number of integrations co-added before readout).

Observations of 4 standard stars from Persson et al. (1998) were obtained on the same night as the 47 Tuc data for calibration purposes.

\subsection{Pre-reduction}

Our pre-reduction, photometry, and calibration procedures are similar to those used by Momany et al. (2003) in a deep nearinfrared study of the globular cluster NGC 6528, using a similar data set and the same observing strategy. We only briefly comment here on the reduction process, and refer the reader to that study for details. In short, for each set of 47 Tuc images, a median background frame was created from the 3 sky frames scaled to a common level, and subtracted from the individual science images. The background-subtracted images were flatfielded and cleaned using master flat-fields, filter-dependent illumination corrections, and bad pixel masks available from the ESO SOFI webpages.

\subsection{Stellar photometry}

Point-spread-function (PSF) fitting stellar photometry was individually carried out on the shallow and deep images using the DAOPHOT II and ALLFRAME (e.g., Stetson 1994). We derived an independent PSF for each image by picking a number of bright and isolated stars; the final PSFs were generated with a "Penny" function and quadratic dependence on the position on the frame. Following the standard ALLFRAME procedure, the 9 deep and 9 shallow images were aligned and combined to obtain a median image, upon which a master list was generated, containing accurate positions of bright and faint stars on the same coordinate system. Using ALLFRAME with that master list, we finally obtained 2 catalogues (separately for the deep and shallow photometry) by matching the $J, H$, and $K_{\mathrm{s}}$ photometry files.

\subsection{Calibration}

Our near-IR calibration techniques are described in some detail by Gullieuszik et al. (2007), and will be only briefly reviewed here. For each standard star, aperture photometry with increasing aperture radii was used to construct growth curves out to a $5 . \prime 2$ radius. The aperture magnitudes of standard stars were normalised to $1 \mathrm{~s}$ exposure time and zero airmass to derive the zero 

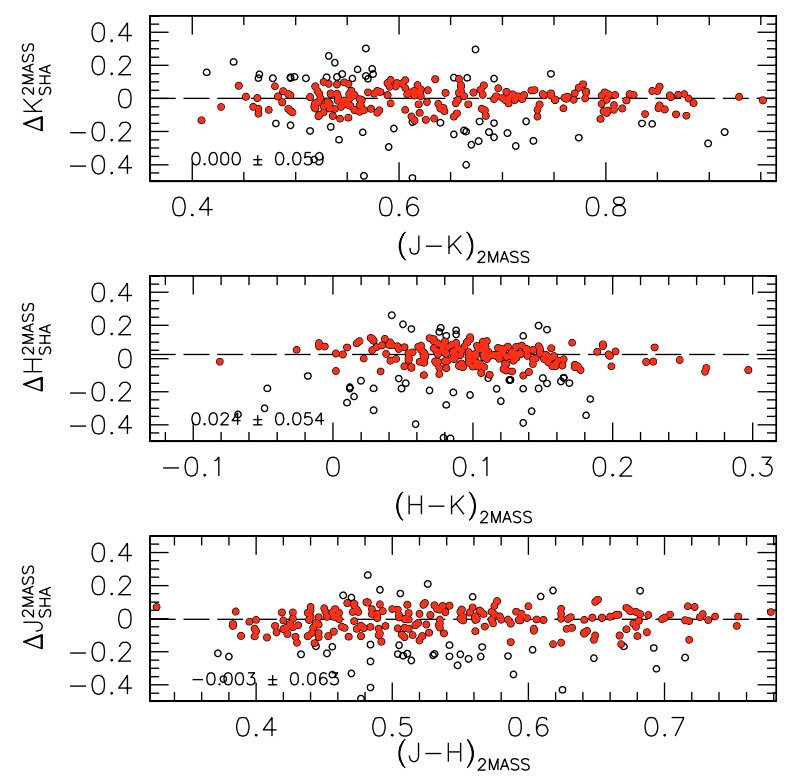

Fig. 1. A comparison between our $J H K_{\mathrm{s}}$ magnitudes and the 2MASS photometry of 47 Tuc. Filled circles (in red in the electronic edition) highlight the $3 \sigma$ clipped stars. The median colour differences are given in each panel along with the standard deviation of the residuals. Note the absence of measurable colour terms.

points and colour terms of the calibration equations (given in Gullieuszik et al. 2007). The calibration equations thus obtained were applied to calibrate the shallow catalogue, after correction of the magnitude scale using aperture photometry of a subset of clean, isolated stars on the best science image in each band. The deep photometry was calibrated by adjusting the instrumental magnitudes onto the zero point of the shallow photometry, using stars in common between the two catalogues.

As a check of our independent photometric calibration, we compare in Fig. 1 our shallow photometry of 47 Tuc with the 2MASS magnitudes for stars in common (Skrutskie et al. 2006). Figure 1 shows that our photometry on the LCO system of Persson et al. (1998) is, for all practical purposes, coincident with the 2MASS system. In fact, no meaningful shifts nor residual colour terms are noticed between the two systems.

\subsection{Photometric errors and incompleteness}

In order to quantify the errors and incompleteness affecting the $K$-band luminosity function of red giant stars, we performed artificial star experiments on our shallow photometry of 47 Tuc. Artificial stars were added to all individual images in the shallow data set in 10 test runs. In each image 470 stars were added, spatially distributed on a grid such that the minimum distance between two artificial stars is $\sim 50$ pixels, which is much larger than the PSF diameter ( $\sim 14$ pixels). This procedure maximizes the number of simulated stars without increasing the crowding of the frames. The input colours and magnitudes of the simulated stars were generated following the RGB ridge line of 47 Tuc between $K_{\mathrm{s}}=15$ and $K_{\mathrm{s}}=8$. The frames including the artificial stars were then reduced, analysed, and calibrated following the same procedures as for the science frames, and the resulting photometry compared with the input catalogue. The difference between the the input and output magnitudes is shown in Fig. 2. The completeness levels, estimated by comparing the number of retrieved stars to the total number of simulated stars, are shown in Fig. 3. Our photometry is clearly complete in the magnitude
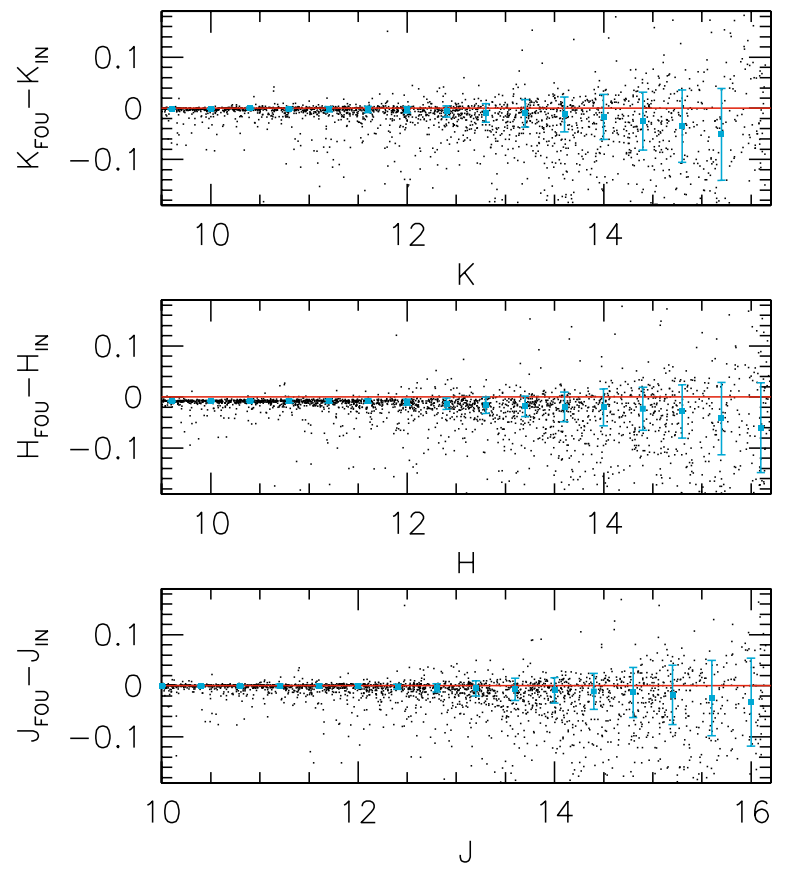

Fig. 2. The photometric errors derived from artificial star experiments on the shallow images, plotted as a function of the magnitude of the retrieved stars. Small dots are the differences between the measured and input magnitudes of artificial stars. The filled squares with the error bars represent the mean and standard deviation of the error distribution in 0.5 mag bins.
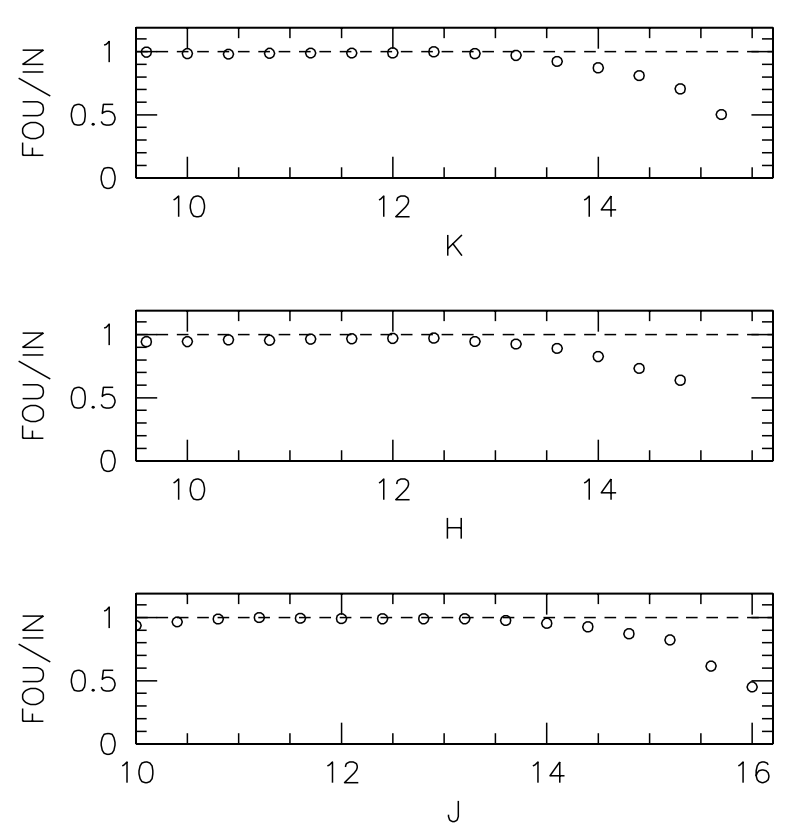

Fig. 3. The completeness of our shallow data as measured from the artificial star experiments. Note the incompleteness in the photometry of bright stars close to saturation.

range $9.5<K_{\mathrm{s}}<13$. The completeness drops smoothly at $K_{\mathrm{s}} \sim 13$, reflecting the loss of faint stars. The completeness of our photometry is also limited on the bright side by saturation $\left(K_{\mathrm{s}} \sim 9.5\right)$. The number of input artificial stars in each magnitude bin is at least 200, which implies for the less complete (faintest) bins a 0.05 formal Poisson uncertainty on the measured completeness. 


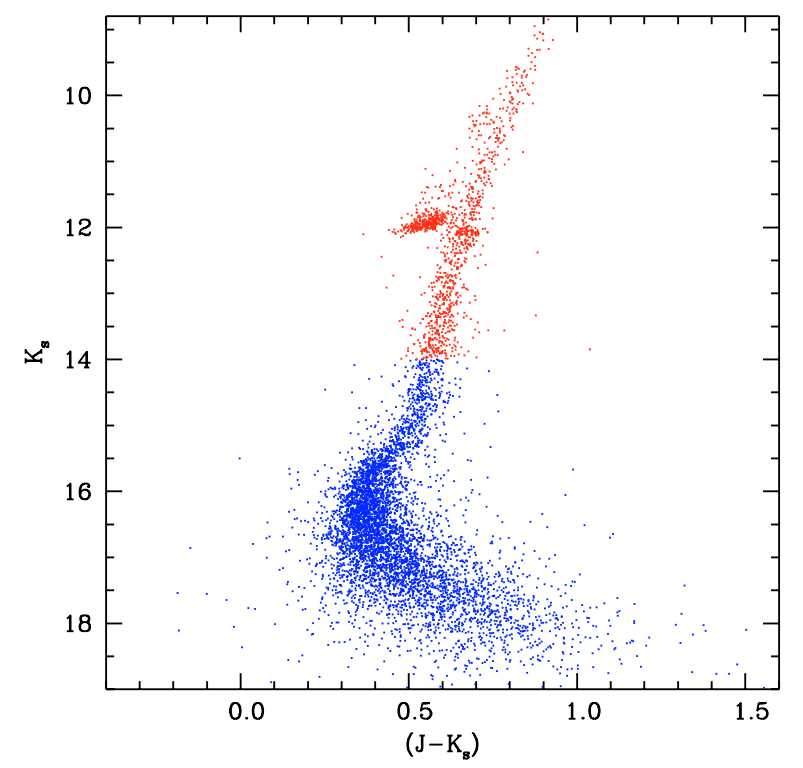

Fig. 4. $K_{\mathrm{s}}-\left(J-K_{\mathrm{s}}\right)$ CMD of 47 Tuc. Stars brighter than $K_{\mathrm{s}}=14$ are taken from the shallow photometry (red in the electronic edition) while fainter stars are taken from the deep catalogue.

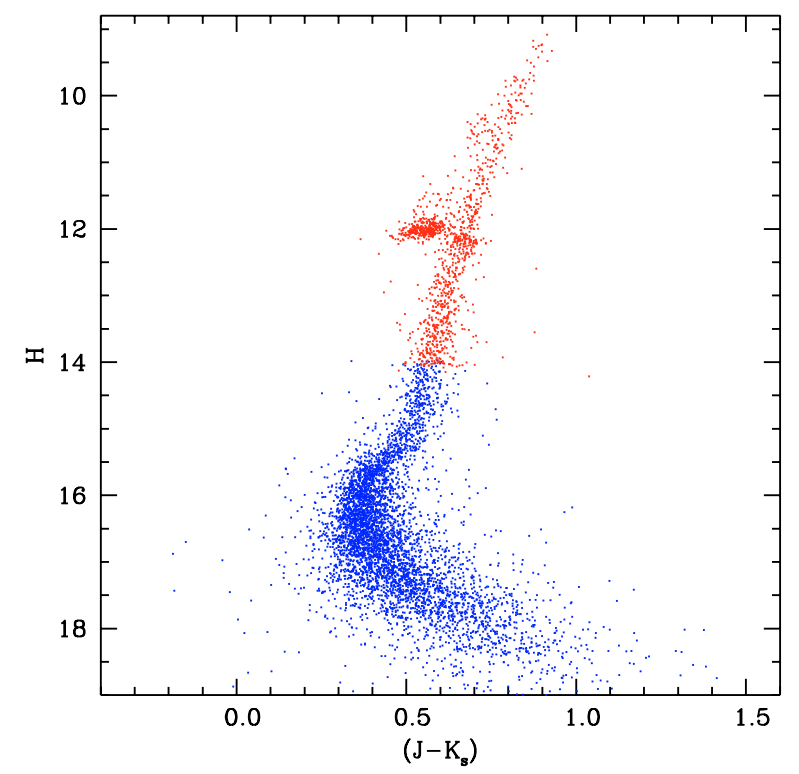

Fig. 5. Same as Fig. 4, but for the $H-\left(J-K_{\mathrm{s}}\right)$ CMD.

\section{Results}

\subsection{Colour-magnitude diagrams}

The CMDs of 47 Tuc obtained from our catalogues are shown in Figs. 4 and 5. These are the deepest near-infrared CMDs published so far for 47 Tuc, reaching $\sim 2$ mag below the mainsequence turnoff in the $K_{\mathrm{s}}$ band. Saturation prevented photometry of the brightest RGB stars near the RGB tip; however these have been discussed in the literature (see, e.g., Valenti et al. 2004a). For both diagrams, we have plotted stars having $\left|\operatorname{sharp}_{K_{\mathrm{s}}}\right|<0.05$.

The width of the RGB is consistent with the photometric errors shown in Fig. 2. The MS is also quite broad, due to the increasingly large photometric error of the deep catalogue near $K_{\mathrm{s}} \sim 17$. When attempting to determine a fiducial line to use for the determination of the turnoff position, we found that colour

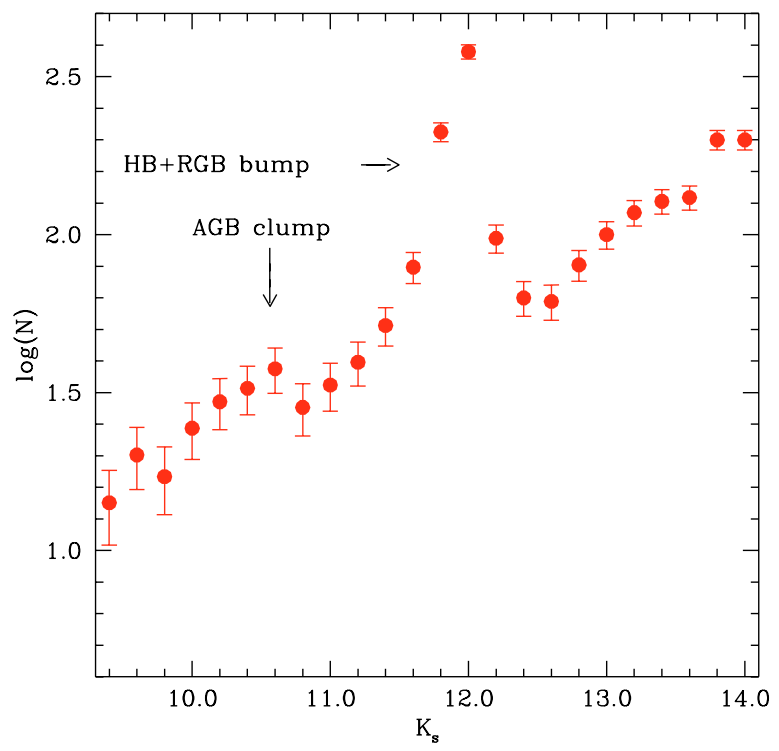

Fig. 6. Observed RGB $K_{\mathrm{s}}$ differential luminosity function ( 0.2 mag bin size). Error bars display the Poisson error on the star counts in each individual bin. The location of the AGB clump, the HB and the RGB bump is marked.

histograms along the MS only show a broad peak in their distribution. Due to the impossibility of defining the exact TO location with high precision, we prefer to use the thickness of the subgiant branch (SGB) to constrain the age from comparisons with theoretical isochrones.

\subsection{The luminosity function}

Figure 6 displays the $K_{\mathrm{s}}$ differential luminosity function (LF star counts, as a function of the $K_{\mathrm{s}}$ magnitude) of all RGB stars and He-burning stars obtained from our photometry. Two local peaks in the star counts are clearly visible. The fainter peak is the more pronounced, and is mainly due to HB stars; the brighter peak is the asymptotic giant branch (AGB) clump, located at $K_{\mathrm{S}} \sim 10.5$, and visible also in the CMDs of Figs. 4 and 5.

The displayed LF does not show the presence of the RGB bump, which is in fact located at a brightness that overlaps with the HB. To determine its exact position, we have selected the RGB stars populating our CMD as shown in Fig. 7. The RGB sequence is well delineated and allows for a clear separation from $\mathrm{HB}$ and $\mathrm{AGB}$ objects. Both differential and cumulative (number of stars brighter than $K_{\mathrm{s}}$, as a function of $K_{\mathrm{s}}$ ) LF of the RGB stars have been computed (Fig. 8). A peak in the differential LF and a slope change in the cumulative LF (Fusi Pecci et al. 1990) mark the position of the bump. Our final determination of the magnitude of the RGB bump in 47 Tuc in shown in Fig. 8. The RGB bump location was estimated by measuring the median of the unbinned data around the RGB-bump peak. We find $K_{\mathrm{s}}^{\text {bump }}=12.047$ and $J^{\text {bump }}=12.71$. An internal measurement error $\sim 0.02$ mag was obtained by varying the magnitude interval used for computing the median (this is more conservative than, e.g., using the formal error of a Gaussian fit to the peak). The total uncertainty on the RGB bump location, including a 0.02 mag error on the photometric zero points, is $0.03 \mathrm{mag}$. Cho \& Lee (2002) determined from 2MASS data $K_{\mathrm{s}}^{\text {bump }}=12.10 \pm 0.10$, in agreement within the errors with our estimate. The same value (but with an error bar of $\pm 0.05 \mathrm{mag}$ ) is obtained from the results 


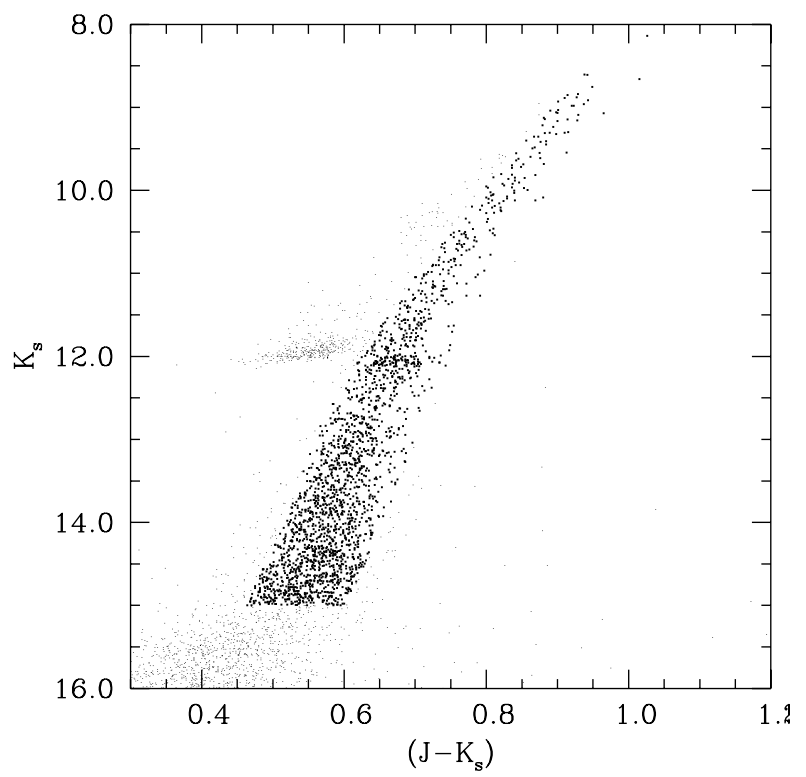

Fig. 7. $K_{\mathrm{s}}-\left(J-K_{\mathrm{s}}\right)$ CMD of 47 Tuc showing the RGB star selection (darker points).

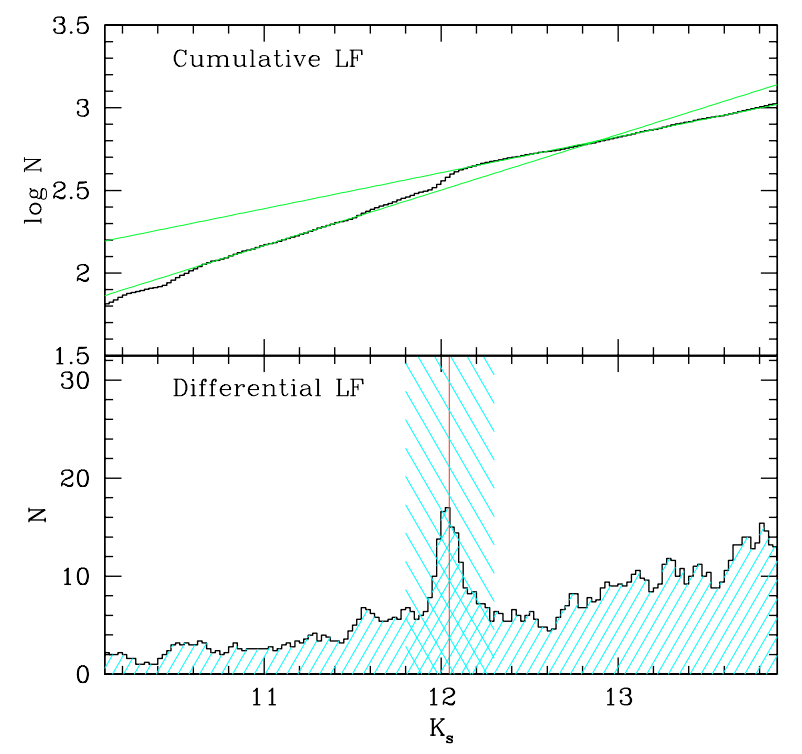

Fig. 8. Measurement of the $K_{\mathrm{s}}$ magnitude of the RGB bump in 47 Tuc. Lower panel: differential luminosity function of RGB stars. The hatched region outlines the magnitude interval used to measure the bump level. A vertical line marks the median magnitude of stars in the bump. Upper panel: cumulative LF of RGB stars. Note the clear jump and change in the LF slope across the bump location.

of Ferraro et al. (2000), transferred to the 2MASS system using Valenti et al. (2004b).

\section{Comparison with theoretical isochrones}

To compare theory with the observed cluster CMDs we made use of the extensive BaSTI database of stellar models and isochrones (computed for both scaled solar and $\alpha$-enhanced heavy element mixtures) described in Pietrinferni et al. (2004, 2006).

More specifically, we used the $\alpha$-enhanced isochrones $(\langle[\alpha / \mathrm{Fe}]\rangle=0.4$, as in Salaris \& Weiss 1998, and in accordance with the value $[\alpha / \mathrm{Fe}] \sim 0.3$ measured by Carretta et al. 2004) computed with the Reimers (1975) mass loss parameter $\eta=0.4$ (although the choice of $\eta$ is not relevant for the results of our analysis) plus $\mathrm{HB}$ models for $[\mathrm{Fe} / \mathrm{H}]=-0.7$, a value that agrees with spectroscopic estimates of 47 Tuc iron (Zinn \& West 1984; Carretta \& Gratton 1997; Kraft \& Ivans 2003; Carretta et al. 2004). An iron content $[\mathrm{Fe} / \mathrm{H}]=-0.7$ corresponds to the isochrones with $Z=0.008$ and $Y=0.256$ in the BASTI database ${ }^{1}$. The adopted initial He abundance is in line with the estimates of Salaris et al. (2004a) based on the $R$ parameter.

We employed the BaSTI isochrones in the $L-T_{\text {eff }}$ plane, transformed to the 2MASS system using the bolometric corrections by Bonatto et al. (2004) based on the methods discussed by Girardi et al. (2000).

\subsection{Distance determination}

We have determined the distance to 47 Tuc from the adopted theoretical models using the HB part of our near-IR CMDs. HB stars are traditionally used to determine globular cluster distances from theoretical models/isochrones, given that their brightness is unaffected by the exact value of the cluster age; moreover, in our case, the lower MS (that can also be used as distance indicator) is affected by excessively large photometric errors and cannot help constrain the distance. It is interesting to notice from Figs. 4 and 5 that the HB is not really horizontal in these photometric filters, not even for red HB morphologies, due to the trend of the near-IR bolometric corrections with $T_{\text {eff }}$. This means that the HB mass distribution affects not only the colour, but also the brightness of this part of the CMD, even for red morphologies.

The cluster distance has been determined by producing synthetic HBs (following the technique pioneered by Rood 1973) and comparing separately the number distribution of objects with the $J, H$ and $K_{\mathrm{s}}$ magnitudes to the observed counterpart, using Kolmogorov-Smirnov (KS) tests. We did not use the HB portion of our adopted $\eta=0.4$ isochrones, because they would not produce extended HBs (no mass dispersion along the HB) and probably would not match the mean mass evolving along the HB of this specific cluster.

More in detail, we first selected as genuine HB stars, all observed objects with $10.90<K_{\mathrm{s}}<12.25$ and $\left(J-K_{\mathrm{S}}\right)<0.62$ (amounting to about $500 \mathrm{HB}$ stars) and then produced a number of synthetic counterparts, as follows. After setting the total number of objects in the synthetic HB simulation to 1000 (the difference with the total number of observed HB stars is not important as long as we use a KS test to compare the two number distributions as a function of the brightness), we started randomly selecting a value of the stellar mass $M_{\mathrm{HB}}$ from a Gaussian distribution centred around a value $\langle M\rangle$, with $1 \sigma$ dispersion $\sigma(\langle M\rangle)$. Both $\langle M\rangle$ and $\sigma(\langle M\rangle)$ are free parameters to be fixed at the start of the simulation; it is well known (see, e.g., Rood 1973) that $\langle M\rangle$ determines the mean colour of the $\mathrm{HB}$ and $\sigma(\langle M\rangle)$ the colour extension around this mean value. In case of the $J H K_{\mathrm{s}}$ filters, as already remarked, the HB is not really horizontal, hence $\langle M\rangle$ and $\sigma(\langle M\rangle)$ also affect the brightness range covered by the HB stars, especially in the $K_{\mathrm{s}}$ filter. After $M_{\mathrm{HB}}$ is determined, a value for the time $t$ since the object arrived on the HB has to be chosen. We determined $t$ by employing a flat probability distribution from zero until $t_{\mathrm{HB}}$, where $t_{\mathrm{HB}}$ is the time spent

1 We employ the most recent $\alpha$-enhanced models in the BaSTI database, recomputed with the low-temperature opacities by Ferguson et al. (2005). More details can be found at http://www . te.astro.it/BASTI/readme.php 
in the HB phase, which is practically constant for the $M_{\mathrm{HB}}$ range typical of clusters with red HBs. The underlying assumption is that stars are being fed onto the HB at a constant rate.

Once $M_{\mathrm{HB}}$ and $t$ are fixed, we interpolated among the BaSTI grid of HB models with the same chemical composition as our reference isochrones $([\mathrm{Fe} / \mathrm{H}]=-0.7,[\alpha / \mathrm{Fe}]=0.4)$, to determine the absolute $J H K_{\mathrm{s}}$ magnitudes of the synthetic HB object; the HB models were computed from an He-core mass and surface $\mathrm{He}$ abundance derived from a progenitor that reaches the RGB tip at an age of 12-13 Gyr (the exact value of the progenitor age is, however, not relevant, as long as it is above a few Gyr). These magnitudes are then perturbed by adding a distance modulus $(m-M)_{0}$ (the third free parameter to be fixed at the start of the simulation) and a Gaussian random error with $1 \sigma$ dispersion, consistent with the values obtained from the artificial star experiments (typical errors of the order of $0.01 \mathrm{mag}$ or less, in all filters). We used $E(B-V)=0.04$ and the extinction law by Rieke $\&$ Lebofsky $(1985)$ to transform $(m-M)_{0}$ into the appropriate apparent distance modulus in $J, H$ and $K_{\mathrm{s}}$. Due to the low reddening of 47 Tuc, the extinction in the near-infrared filters is extremely small, between 0.01 and 0.03 mag. Finally, the synthetic objects are accepted or rejected by simulating the trend of completeness fractions with the relevant filter. The level of completeness in the HB magnitude range is, however, practically always about $100 \%$ (see Fig. 3 ) so that this correction is essentially negligible. The procedure is repeated until the HB is populated by 1000 objects.

The resulting object number distributions with the $J, H$ and $K_{\mathrm{S}}$ magnitudes are then separately compared to the observed counterpart by means of a KS test, for each choice of $\langle M\rangle$, $\sigma(\langle M\rangle)$ and $(m-M)_{0}$. We accept all combinations of $\langle M\rangle$, $\sigma(\langle M\rangle)$ and $(m-M)_{0}$ values giving a probability $P<95 \%$ that observed and theoretical number distributions are different. We find a consistent set of solutions that satisfies the condition on $P$ simultaneously for all three photometric bands. We explored with the KS test the following $(m-M)_{0},\langle M\rangle$ and $\sigma(\langle M\rangle)$ ranges: $13.10 \leq(m-M)_{0} \leq 13.30,0.61 \leq\langle M\rangle / M_{\odot} \leq 0.68$, $0.0 \leq \sigma(\langle M\rangle) / M_{\odot} \leq 0.03$. These have been selected on the basis of preliminary simulations, showing how combinations of parameters outside these boundaries produce magnitude distributions macroscopically different from the observed ones. As an example, just a simple comparison of the location of the theoretical zero age horizontal branch (ZAHB, corresponding to the lower envelope of the observed HB distribution) with the observed CMDs displays a total disagreement for $(m-M)_{0}<13.10$ or $(m-M)_{0}>13.30$.

Figure 9 displays the region in the $(m-M)_{0}-\langle M\rangle$ plane where $P<95 \%$ for all three photometric filters. The minimum values of $P$ are of the order of $50-55 \%$.

The resulting distance modulus is $(m-M)_{0}=13.18 \pm 0.03$, where 13.18 is the central value of the $(m-M)$ range allowed by the condition $P<95 \%$. Considering HB models from the BaSTI database with different $[\mathrm{Fe} / \mathrm{H}]$, we estimate an additional systematic error of $\pm 0.04 \mathrm{mag}$, due to a typical uncertainty of \pm 0.1 dex on $47 \mathrm{Tuc}[\mathrm{Fe} / \mathrm{H}]$ estimates. The \pm 0.02 mag uncertainty on $E(B-V)$ gives a negligible contribution to the systematic error when added in quadrature to the effect of metallicity. To summarize, we obtain from the theoretical models $(m-M)_{0}=$ $13.18 \pm 0.03$ (random $) \pm 0.04$ (systematic). The mean HB mass $\langle M\rangle$ is in the range between 0.65 and $0.66 M_{\odot}$, and the dispersion $\sigma(\langle M\rangle)$ is between 0.010 and $0.012 M_{\odot}$.

Figure 10 displays, as a visual example (we recall that the KS test does not require the data to be binned) a comparison between the observed HB differential luminosity

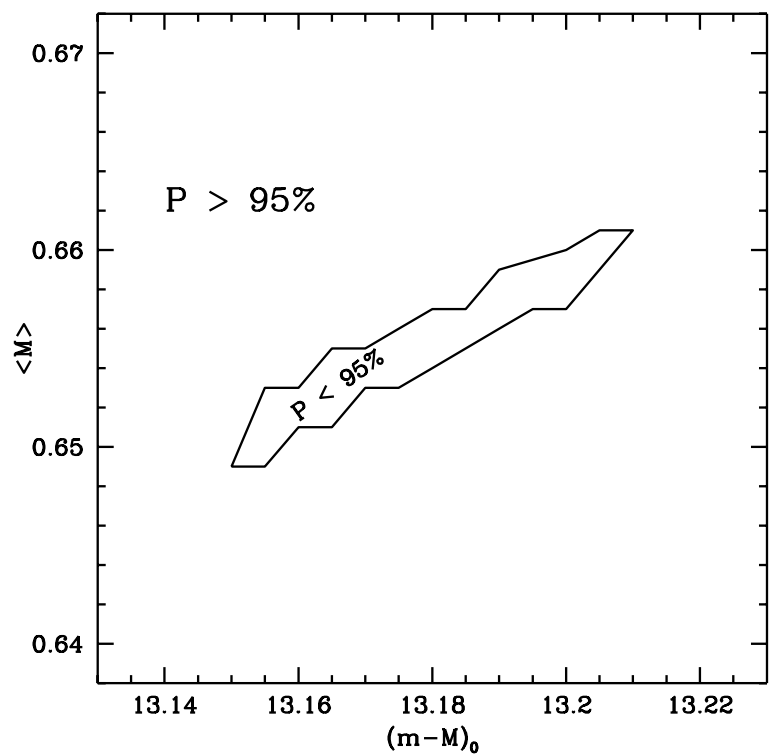

Fig. 9. Region in the $\langle M\rangle-(m-M)_{0}$ parameter space where the KS test provides $P<95 \%$ in all three photometric bands (see text for details).
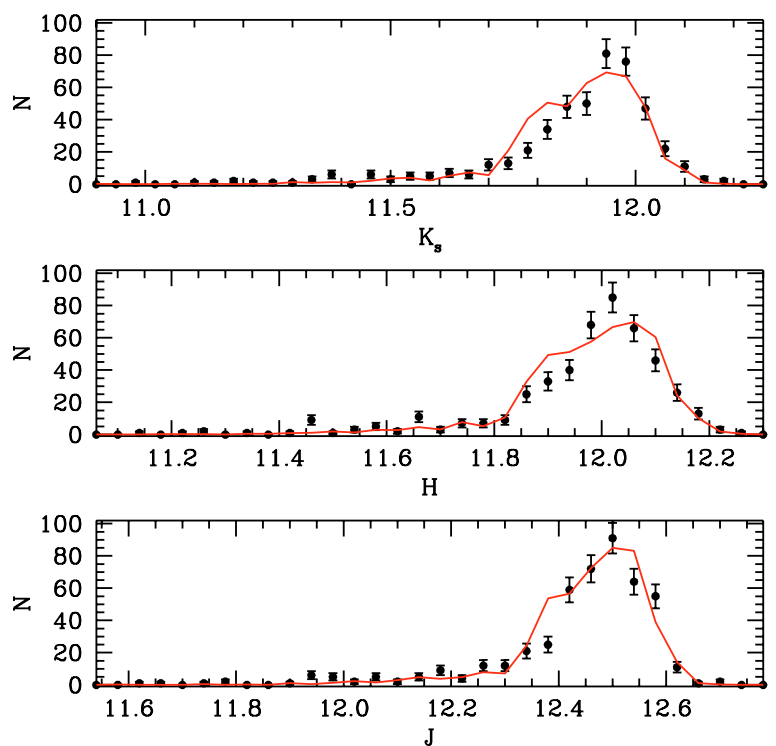

Fig. 10. Observed HB differential luminosity function (filled circles) compared to the $\alpha$-enhanced synthetic counterpart (continuous line) with $[\mathrm{Fe} / \mathrm{H}]=-0.7,\langle M\rangle=0.655 M_{\odot}$, and $\sigma(\langle M\rangle)=0.012 M_{\odot}$, assuming $E(B-V)=0.04$ and $(m-M)_{0}=13.18$.

function, and the theoretical counterpart obtained from a simulation with $\langle M\rangle=0.655 M_{\odot}, \sigma(\langle M\rangle)=0.012 M_{\odot}$ and $(m-M)_{0}=$ 13.18 , a combination of parameters that satisfies the condition on $P$. The mean magnitudes of the HB stars in the three photometric filters are reproduced within $0.01 \mathrm{mag}$.

\subsection{CMD and theoretical isochrones}

Adopting the distance derived above, Figs. 11 and 12 compare the observed $K-\left(J-K_{\mathrm{s}}\right)$ and $H-\left(J-K_{\mathrm{s}}\right)$ CMDs with BaSTI $[\mathrm{Fe} / \mathrm{H}]=-0.7,[\alpha / \mathrm{Fe}]=0.4$ theoretical isochrones for ages of 10 and 13 Gyr. The isochrones are computed with a mass loss efficiency parameter $\eta=0.4$, but this does not affect the location of the RGB. 


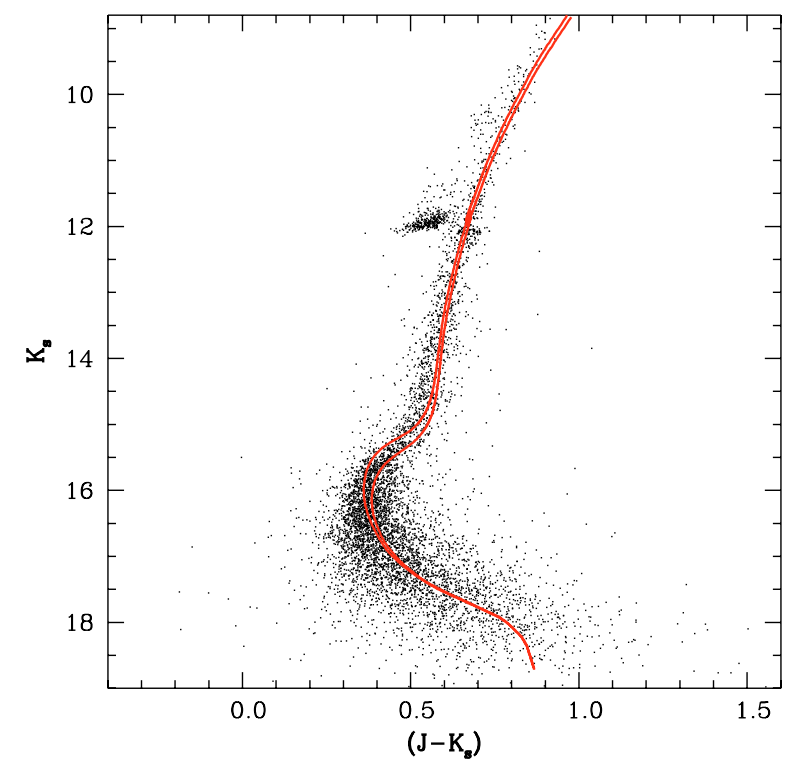

Fig. 11. $K_{\mathrm{s}}-\left(J-K_{\mathrm{s}}\right) \mathrm{CMD}$ of 47 Tuc compared to 10 and 13 Gyr old, $[\mathrm{Fe} / \mathrm{H}]=-0.7 \alpha$-enhanced isochrones from the BaSTI database (in red in the electronic edition). A distance modulus $(m-M)_{0}=13.18$ and reddening $E(B-V)=0.04$ have been applied to the models (see text for details).

The isochrones follow nicely the sequences described by the observed CMDs, although one has to take into account that the broad MS does not put strong constraints on the accuracy of MS theoretical colours. Some mismatch (models too red) appears at the base of the RGB but, starting more than a magnitude below the bump region, the models reproduce nicely the observed RGB sequence. As discussed before, we cannot determine with any degree of accuracy the position of the TO in the CMD, therefore we use the vertical thickness of the SGB to put some - admittedly weak - constraints on the cluster age. One can see more clearly from the $H-\left(J-K_{\mathrm{s}}\right) \mathrm{CMD}$ that the SGB of the $13 \mathrm{Gyr}$ isochrone runs approximately along the faint end of the observed SGB, whereas the $10 \mathrm{Gyr}$ isochrone is near the upper envelope of the observed SGB. A realistic estimate of the cluster age is therefore most probably contained between these two limits, and this is consistent with independent recent determinations of 12.5 \pm 0.5 Gyr (Liu \& Chaboyer 2000), 10.9 \pm 1.4 Gyr (Salaris et al. 2004b) and 11.5 $\pm 0.8 \mathrm{Gyr}$ (VandenBerg 2000).

It is important to notice that the initial MS mass of the stars at the RGB tip, for ages between 10 and $13 \mathrm{Gyr}$, is in the range between $\sim 0.95$ and $\sim 0.89 M_{\odot}$. A mean mass of $\sim 0.65 M_{\odot}$ for the objects still evolving along the HB means that their RGB progenitors have lost $0.24-0.30 M_{\odot}$ during their RGB ascent. This amount of mass loss corresponds to $\eta \sim 0.5$ in the Reimers formula.

\section{The number of stars along the RGB}

Schiavon et al. (2002, hereafter S02) discovered a worrying discrepancy between the number of RGB stars above the level of the $\mathrm{HB}$ and the theoretical predictions from independent theoretical isochrones by both Girardi et al. (2000) and Salaris \& Weiss (1998). Actually S02 employed $\alpha$-enhanced isochrones computed with the same code and input physics as in Salaris \& Weiss (1998), but including atomic diffusion. Diffusion does not however alter the shape of the RGB differential luminosity function. After theoretical star counts along the RGB are

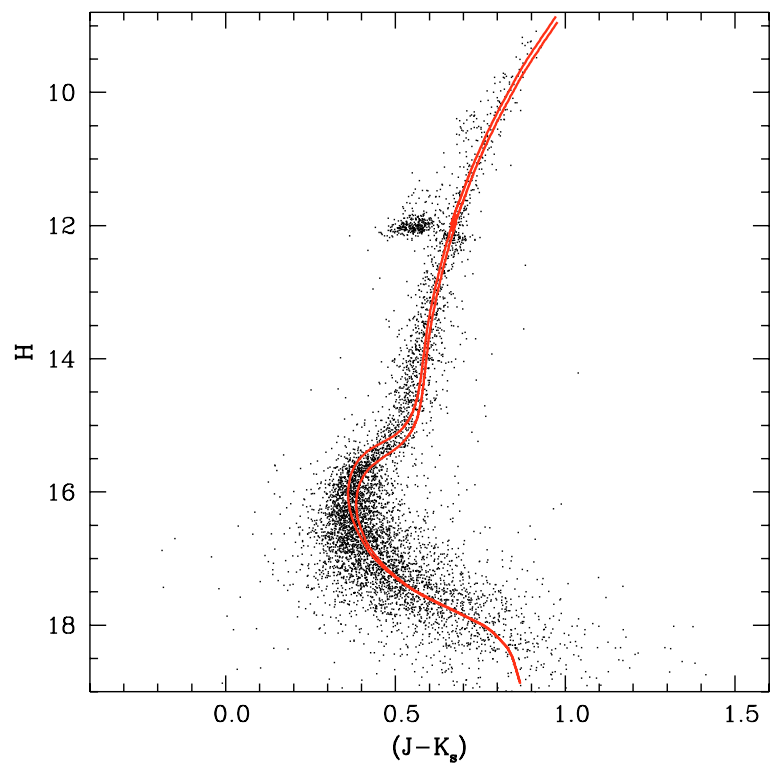

Fig. 12. As in Fig. 11, but for the $H-\left(J-K_{\mathrm{s}}\right)$ CMD.

properly normalised, they agree with observations at luminosities below the HB level, whereas they predict about a factor of 2 fewer stars at luminosities above the HB. This means that, according to S02, the theoretical slope of the RGB differential luminosity function is inconsistent with the one observed in 47 Tuc. As discussed in detail by S02, this discrepancy affects spectroscopic ages obtained from $\mathrm{H}_{\beta}$ and $\mathrm{H}_{\gamma}$ absorption feature indices, because RGB stars contribute a significant fraction of the continuum flux of old stellar populations, even in the blue wavelength range of the age-sensitive absorption features.

Using our new near-IR data we investigate here this very important issue, and test whether our adopted isochrones are able to reproduce the observed number of RGB stars both below and above the HB level. We show here the results obtained from RGB star counts in the $K_{\mathrm{s}}$ band. We checked that the same results are obtained when considering star counts in the $J$ and $H$ photometric filters. An important advantage of using near-infrared filters is that the bolometric corrections are largely independent of metallicity and the metal mixture (Cassisi et al. 2004). Therefore, the fact that we are using colour transformations to the 2MASS system based on scaled solar model atmospheres, whereas the underlying theoretical isochrones are $\alpha$-enhanced, does not affect our analysis at all.

The RGB star selection from the observed $K_{\mathrm{s}}-\left(J-K_{\mathrm{s}}\right)$ CMD is the same as discussed in Sect. 3. The differential luminosity function (LF) for the RGB stars in the shallow catalogue of 47 Tuc - with a bin size of 0.2 mag, with and without corrections for the completeness - together with the completeness fraction along the RGB, is displayed in Fig. 13. Since the completeness is always above $90 \%$ for $K_{\mathrm{s}}$ between $\sim 9.5$ and $\sim 14 \mathrm{mag}$, we will concentrate our analysis in this region. A preliminary comparison of the observed LF with theory is performed in Fig. 14. There is an important point to consider when comparing S02 and our analysis. In S02, the comparison of the observed LF with theory was performed by normalising the theoretical LF to the observed one at the TO region. Instead, we have chosen to normalise the theoretical LF to the total number of stars (corrected for the small effect of completeness) in the magnitude range $12.4<K_{\mathrm{S}}<13.2$. Given that $\mathrm{S} 02$ found a discrepancy between theory and observations only along the bright part of the RGB (approximately above the bump+HB region) we should, in 


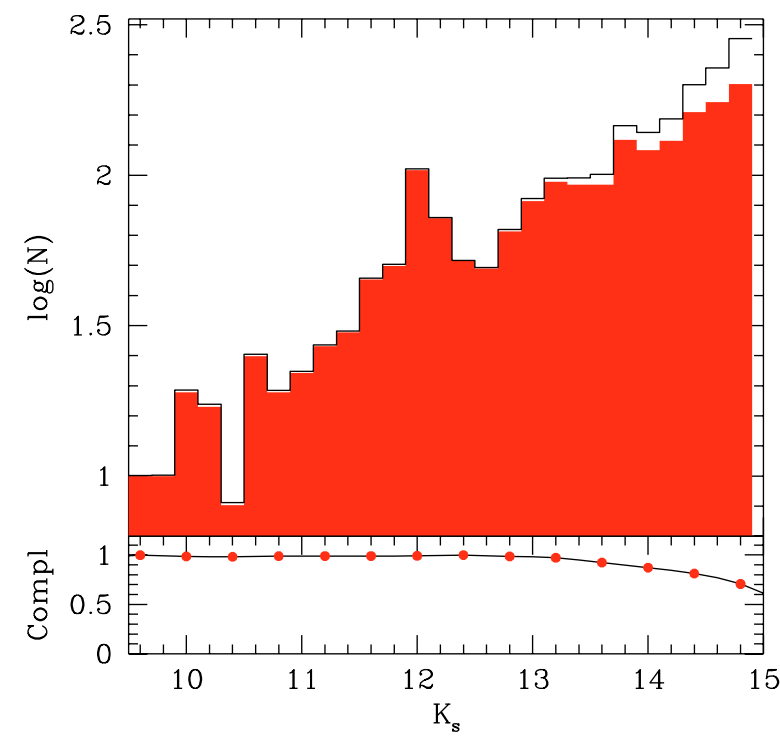

Fig. 13. The logarithm of the observed RGB differential luminosity function in $K_{\mathrm{s}}$. The shaded area highlights the effect of neglecting the correction for the completeness. The lower panel shows the value of the completeness fraction as a function of $K_{\mathrm{s}}$.

principle, be able to find the same discrepancy, even with our normalisation to the number of RGB stars along a section of the faint RGB sequence. Also, the number of stars populating our LF should be approximately the same as for S02 LF. As a reference, S02 give a number of $62 \mathrm{AGB}+\mathrm{RGB}$ objects in their LF, in the range $12.8<V<13.2$. Taking advantage of the 47 Tuc mean $K-(V-K)$ relations by Ferraro et al. (2000) - again transferred to the 2MASS system using Valenti et al. (2004b) - and their adopted distance moduli and reddenings, we have been able to estimate the number of objects in the $K_{\mathrm{s}}$ magnitude range corresponding to the $V$ interval given by S02. We find 69 objects, approximately the same as the counterpart in the S02 luminosity function.

Figure 14 shows, at a glance, a general agreement between theory and observations, without a significant offset above the RGB bump region, contrary to the results by S02. It is interesting to notice that the RGB bump is clearly fainter than the models. We will come back to this point later in this section.

To avoid binning the data, and to include easily in a more quantitative comparison the effect of the - albeit small - photometric errors and completeness fraction, we again made use of Monte Carlo (MC) simulations and KS tests in the analysis that follows. Our main aim is to test whether the star number distribution predicted by the theoretical isochrones along the whole observed RGB sequence is consistent with the observed counterpart. To this purpose - and in the same vein as the HB distance estimate discussed before - we have produced synthetic samples of RGB stars with a MC technique applied to the theoretical isochrones. A theoretical isochrone of a given metallicity and age is selected, and the effect of extinction and distance modulus is added to the absolute $K$ magnitudes. The Salpeter (1955) Initial Mass Function (IMF) has then been used to draw randomly stellar masses between the lower and upper mass limits of the RGB population; the precise choice of the IMF exponent does not affect the results, given the very narrow mass range of the objects populating the RGB. The appropriate $K_{\mathrm{s}}$ magnitude is then assigned to each mass, by interpolating among neighbouring points along the isochrone. These $K_{\mathrm{s}}$ magnitudes are then perturbed by a Gaussian $1 \sigma$ photometric error,

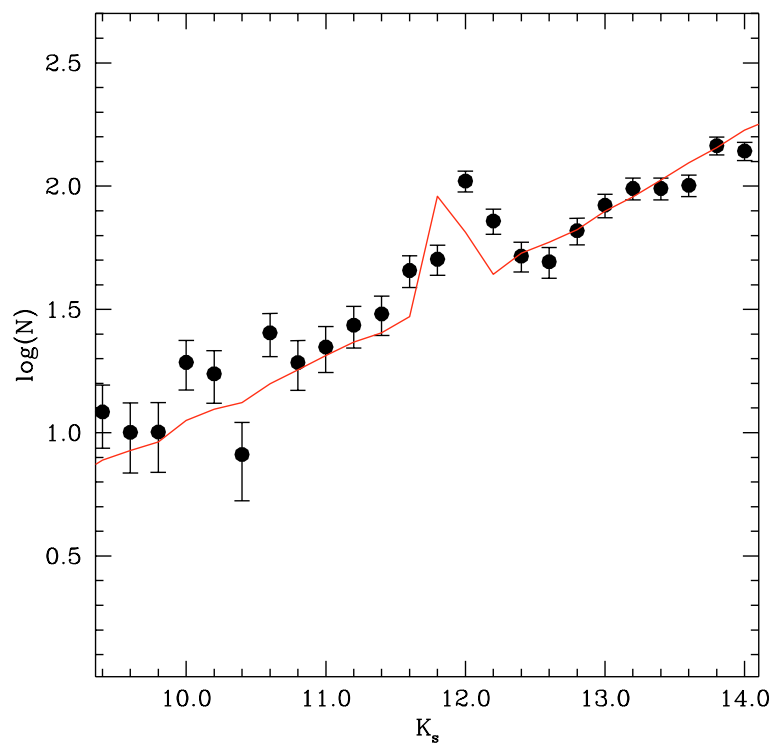

Fig. 14. Logarithm of the observed RGB luminosity function (filled circles - completeness corrections applied) with a 0.2 mag bin-size, compared to the theoretical counterpart for $[\mathrm{Fe} / \mathrm{H}]=-0.7$, an age of $11 \mathrm{Gyr}$ and $(m-M)_{0}=13.18$. The theoretical LF has been normalised to the observed number of stars in the range $12.4<K_{\mathrm{s}}<13.2$. Error bars due to Poisson statistics are also displayed.

consistent with the values obtained from the artificial star experiments (typical errors of the order of $0.01 \mathrm{mag}$ or less, in all filters). Finally, the synthetic objects are accepted or rejected by simulating the trend of completeness fractions with $K_{\mathrm{s}}$. The total number of stars drawn in the simulation is very large, of the order of $10^{5}$ objects, to avoid statistical fluctuations in the theoretical star counts. The difference with the total number of observed RGB stars is not important as long as we use a KS test to compare unbinned predicted and observed number distributions as a function of $K_{\mathrm{s}}$. We accept the existence of a statistically significant difference between theory and observations whenever we obtain a probability $P>95 \%$ that observed and theoretical number distributions are different.

Our reference $\mathrm{MC}$ simulation employed $[\mathrm{Fe} / \mathrm{H}]=-0.7$, an age of $11.0 \mathrm{Gyr}$ and $(m-M)_{0}=13.18$. If we compare the LFs for the whole RGB with $K_{\mathrm{s}}<14.0$, we obtain $P \sim 100 \%$, i.e., a very significant discrepancy between theory and observations. Given the general agreement displayed by Fig. 14, we suspected that this value of $P$ could be mainly due to the discrepant luminosity of the bump, and we repeated our KS-test analysis by dividing the RGB into three magnitude ranges. In the range $9.5 \leq K_{\mathrm{s}} \leq 11.5$, above the observed RGB bump, $P$ is always smaller than $\sim 80 \%$. Below the observed bump, for $K_{\mathrm{s}}>12.6, P$ is always smaller than $30 \%$. The bump region, $11.5 \leq K_{\mathrm{s}} \leq 12.6$ shows $P \sim 100 \%$.

To show this discrepancy even more clearly, Fig. 15 compares the observed and theoretical LF around the bump region, with a $0.04 \mathrm{mag}$ bin-size. The theoretical LF is normalised as in Fig. 14. The different luminosity of the bump is evident; the difference between the average $K_{\mathrm{s}}$ of the bump in the observed and theoretical LF amounts to $\Delta K_{\mathrm{s}} \sim 0.15 \mathrm{mag}$, the theoretical bump being brighter.

In a second set of tests we have considered the combined number distribution of RGB plus early-AGB stars with $K_{\mathrm{s}}<$ 10.9 , that is, the total number of stars above the HB (the HB theoretical star distribution has been already forced to match the observed one in our determination of the cluster distance) that 


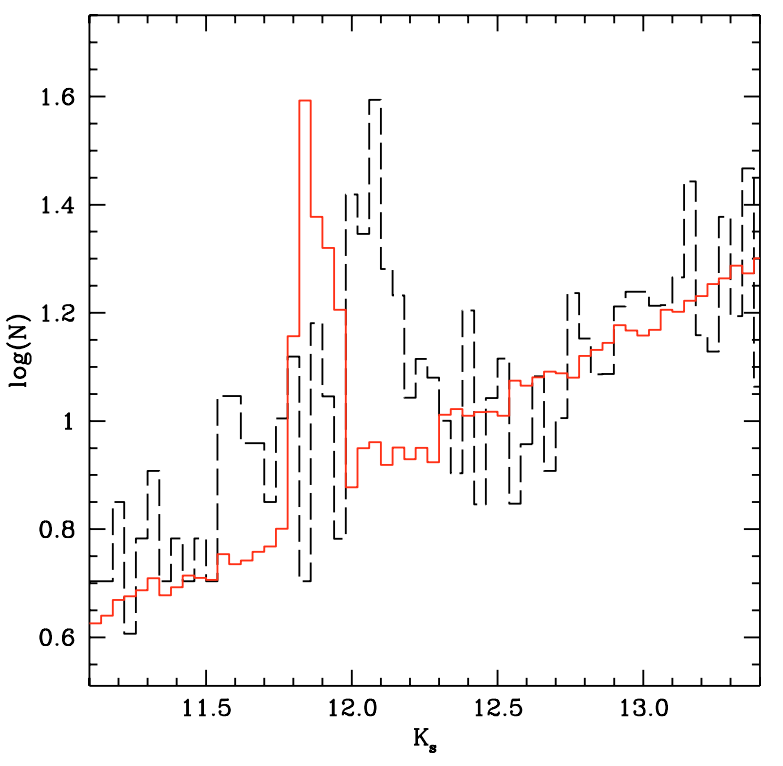

Fig. 15. Observed LF for RGB stars in the bump region (dashed line) compared to the theoretical counterpart (solid line, in red in the electronic edition) for $[\mathrm{Fe} / \mathrm{H}]=-0.7$, an age of $11 \mathrm{Gyr},(m-M)_{0}=13.18$ and a 0.04 mag bin size.

also contains objects belonging to the AGB-clump. We include in this analysis the AGB part of the $\eta=0.4$ isochrones, given that the AGB region is unaffected by the exact value of the evolving mass, for the mass range typical of red HBs. A comparison of theory with observations using the KS test and the methods described before provides $P \sim 20 \%$.

Before closing this section, we also investigate the discrepancy between predicted and observed values of the quantity $R_{\text {bump }}$, i.e., the ratio between star counts across the RGB bump and fainter RGB stars, discussed by Bono et al. (2001). More specifically, $R_{\text {bump }}$ has been defined by Bono et al. (2001) in the Johnson $V$-band as the ratio between the number of RGB stars within $V^{\text {bump }} \pm 0.4$ and the number of RGB stars within $V^{\text {bump }}+0.5 \leq V \leq V^{\text {bump }}+1.5$. $R_{\text {bump }}$ relies on star counts that do not depend on the bin size nor on the bump luminosity, and it is a diagnostic of the size of the H-profile discontinuity left over by the bottom boundary of the convective envelope at its largest extension.

We have determined an equivalent parameter $R_{\text {bump }}^{K_{\mathrm{s}}}$, defined as the ratio of the number of stars within $K_{\mathrm{s}}^{\text {bump }} \pm 0.4$, to the number of objects within $K_{\mathrm{s}}^{\text {bump }}+0.65 \leq K_{\mathrm{s}} \leq K_{\mathrm{s}}^{\text {bump }}+1.55$. Our data provide $R_{\text {bump }}^{K_{\mathrm{s}}}=0.71 \pm 0.05$. The value predicted from our reference $11 \mathrm{Gyr}$ old, $[\mathrm{Fe} / \mathrm{H}]=-0.7, \alpha$-enhanced isochrone is $R_{\text {bump }}^{K_{\mathrm{s}}}=0.64$, i.e. the discrepancy with observations, is well below the $2 \sigma$ level, much less significant than found by Bono et al. (2001) from an independent photometry in the $B V$ system.

\subsection{Playing with parameters}

On the basis of the results discussed before, we can exclude a significant discrepancy between theoretical and observed trends of star number counts versus $K_{\mathrm{s}}$ along the whole RGB sampled by our data. The same results are obtained considering the number counts as a function of $J$ and $H$ magnitudes. The only discrepancy between theory and observation is the position of the RGB bump. With our derived distance modulus, a $11 \mathrm{Gyr}$

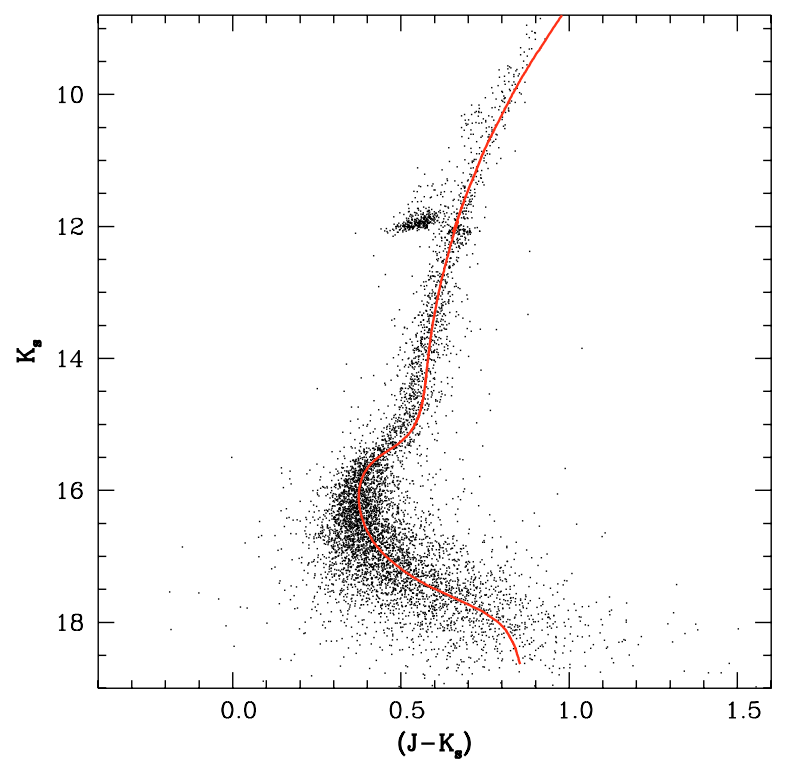

Fig. 16. $K_{\mathrm{s}}-\left(J-K_{\mathrm{s}}\right) \mathrm{CMD}$ of $47 \mathrm{Tuc}$ compared to $13 \mathrm{Gyr}$ old, $[\mathrm{Fe} / \mathrm{H}]=$ $-0.6 \alpha$-enhanced isochrones from the BASTI database (in red in the electronic edition). A distance modulus $(m-M)_{K}=13.14$ and reddening $E(B-V)=0.02$ have been applied to the models (see text for details).

isochrone with $[\mathrm{Fe} / \mathrm{H}]=-0.7$ and $[\alpha / \mathrm{Fe}]=0.4$ displays a RGB-bump about 0.15 mag brighter than observations.

We have performed additional comparisons by varying some parameters within realistic ranges, and studied the effect on the bump luminosity. A decrease of $[\alpha / \mathrm{Fe}]$ from 0.4 to 0.0 (we recall that Carretta et al. 2004 measured $[\alpha / \mathrm{Fe}] \sim 0.3)$ at fixed $[\mathrm{Fe} / \mathrm{H}]$ increases the HB brightness by $0.05 \mathrm{mag}$ in the near-infrared bands. This causes an increase of the distance modulus by the same amount, but the bump brightness increases by 0.24 mag, and therefore the discrepancy between theory and observations is exacerbated. An increase of $[\mathrm{Fe} / \mathrm{H}]$ by 0.1 dex (typical error associated with the spectroscopic determination for this cluster) at fixed $[\alpha / \mathrm{Fe}]$ decreases the distance modulus by $\sim 0.04$, because of a fainter HB, and the bump brightness decreases by 0.12 mag. The net effect is to reduce the size of the discrepancy by half. Changing the age from 11 to 15 Gyr decreases the bump brightness by $0.10 \mathrm{mag}$, keeping the distance modulus unchanged.

As a conclusion, a combination $[\mathrm{Fe} / \mathrm{H}]=-0.6,[\alpha / \mathrm{Fe}]=0.4$ and an age of $\sim 13-14$ Gyr would eliminate the discrepancy between the observed and predicted bump brightness. Figure 16 displays a comparison between the $K_{\mathrm{s}}-\left(J-K_{\mathrm{s}}\right)$ CMD of 47 Tuc and a $13 \mathrm{Gyr}$ isochrone with $[\mathrm{Fe} / \mathrm{H}]=-0.6,[\alpha / \mathrm{Fe}]=0.4$, $(m-M)_{0}=13.14$ and $E(B-V)=0.02$. The quality of the fit is very similar to the case of the $13 \mathrm{Gyr},[\mathrm{Fe} / \mathrm{H}]=-0.7$ models; the reduced reddening is still within the commonly quoted error bars. The value of $R_{\text {bump }}^{K_{\mathrm{s}}}$ obtained from this isochrone is essentially unchanged compared to the $11 \mathrm{Gyr},[\mathrm{Fe} / \mathrm{H}]=-0.7$ case, and it would therefore still be within less than $2 \sigma$ from the observed $R_{\text {bump }}^{K_{\mathrm{s}}}$.

An alternative solution to the problem of the observed bump brightness is to keep the age fixed at $11 \mathrm{Gyr},[\mathrm{Fe} / \mathrm{H}]=-0.7$, $[\alpha / \mathrm{Fe}]=0.4$ and the distance modulus as derived in Sect. 4.1, but introduce some degree of overshooting (as a free parameter) from the bottom of the convective envelopes into the surrounding, formally stable, radiative regions (Alongi et al. 1991). This results in a thicker convective envelope, deeper location of the H-profile discontinuity left over by convection at its 
Table 2. Recent parallax-based distance determinations for 47 Tuc.

\begin{tabular}{llll}
\hline \hline Reference & Method & $(m-M)_{0}$ & $E(B-V)$ \\
\hline Reid (1998) & MS fitting & $13.56 \pm 0.15$ & 0.040 \\
Carretta et al. (2000) & MS fitting & $13.38 \pm 0.09$ & 0.055 \\
Zoccali et al. (2001) & White dwarf fitting & $13.09 \pm 0.14$ & 0.055 \\
Percival et al. (2002) & MS fitting & $13.25_{-0.07}^{+0.06}$ & 0.040 \\
Salaris \& Girardi (2002) & Red clump & $13.19 \pm 0.07$ & 0.055 \\
\hline
\end{tabular}

maximum extension, and a fainter bump. Based on the numerical experiments reported in Cassisi et al. (2002), we estimate that an overshooting by $\sim 0.2 H_{\mathrm{p}}$ beyond the formal Schwarzschild boundary of the convective envelope would eliminate the discrepancy. Such a moderate amount of overshooting would not appreciably affect the predicted value of $R_{\mathrm{bump}}^{K_{\mathrm{S}}}$ compared to the non-overshooting case (Cassisi et al. 2002).

Also, based on the results by Cassisi et al. (1997), one can infer that the inclusion of atomic diffusion would only marginally improve the agreement between predicted and observed bump brightness, but the efficiency of this process in globular cluster stars is still very uncertain (Gratton et al. 2001; Korn et al. 2006).

The effect of the parameter (age, chemical composition) changes discussed before on the comparison of the RGB and AGB star counts - excluding the RGB bump region - is negligible. By repeating the KS tests, we find that the values of $P$ considering only RGB stars above and below the bump, and the combined RGB+AGB objects above the HB, are almost unchanged compared to the reference case of an $11 \mathrm{Gyr}$ isochrone with $[\mathrm{Fe} / \mathrm{H}]=-0.7$ and $[\alpha / \mathrm{Fe}]=0.4$. In any case, $P$ is always well below the $95 \%$ threshold.

We note that the adopted distance is also constrained by the observed location of the AGB bump in 47 Tuc. We found that a change of the distance modulus of the order of 0.10 mag would destroy the agreement of the RGB +AGB LF, because in this case the AGB bump region - that is well reproduced with our reference distance, and is very weakly sensitive to chemical composition and age (Pulone 1992) - would be badly reproduced by the theoretical models.

\section{Summary and conclusions}

We have presented the deepest to date near-IR photometry of 47 Tuc, a cluster central to studies of Galaxy formation, and a testbed for theoretical models, distance determination and extragalactic age-dating techniques.

We have derived the cluster distance by fitting synthetic HB models to the observed CMD, in the $J, H$ and $K_{\mathrm{s}}$ bands, using a method based on the KS test. Assuming $E(B-V)=$ $0.04 \pm 0.02$, we have obtained a distance modulus $(m-M)_{0}=$ $13.18 \pm 0.03$ (random $) \pm 0.04$ (systematic) . Once the HB star distribution is matched by synthetic HB models, the observed brightness of the AGB bump is also well reproduced by theory.

Our derived distance is almost identical to the value $(m-$ $M)_{0}=13.19 \pm 0.07$ obtained by Salaris \& Girardi (2002), who applied their population corrections to the HIPPARCOS $K_{\mathrm{s}}$ magnitude of the solar neighbourhood Red Clump, and used 47 Tuc 2MASS data analysed by Grocholski \& Sarajedini (2002). Table 2 summarises the most recent determinations of 47 Tuc distance modulus, obtained from parallax-based methods $^{2}$. The distance modulus of 47 Tuc from optical data has been

${ }^{2}$ Each of these methods also employs, to different degrees, results from theoretical models, but only in a differential way. recently discussed by Percival et al. (2002) and Zoccali et al. (2001). Percival et al. revised previous MS-fitting determinations and, using Kaluzny et al. (1998) recalibrated data, concluded that the dereddened modulus is $(m-M)_{0}=13.25_{-0.07}^{+0.06}$, shorter than most of the previous results (13.38 from Carretta et al. 2000; 13.56 from Reid 1998). Zoccali et al. (2001) derived the distance to 47 Tuc from the white dwarfs cooling sequence compared to the local field white dwarfs measured with the same instrument. This method is independent of the HB models as well as of the local subdwarfs. They obtained $(m-M)_{0}=13.09$ assuming $E(B-V)=0.055$, shorter than the distance of Carretta et al., but compatible - within the $1 \sigma$ errors - with that found by Percival et al. (2002). Notice also that the slightly different $E(B-V)$ values adopted by different authors do not substantially modify the comparison of their results. The theoretical HB-fitting distance we obtain in this work confirms the relatively "short distance" for 47 Tuc found by Zoccali et al. (2001) and Percival et al. (2002).

As a byproduct of the synthetic HB fitting, we obtain a mean HB star mass $\langle M\rangle$ in the range between 0.65 and $0.66 M_{\odot}$, and the dispersion $\sigma(\langle M\rangle)$ is between 0.010 and $0.012 M_{\odot}$.

Constraints on the cluster age have been obtained by fitting theoretical isochrones to the observed CMD, employing the distance obtained from the synthetic HB models. We obtain an age in the range between $\sim 10$ and $\sim 13 \mathrm{Gyr}$, in agreement with previous independent estimates.

We have also investigated in detail the level of agreement between the theoretical and observed LF of the RGB (and early-AGB) stars, to verify previous claims of a significant discrepancy between theory and observations in 47 Tuc. Using again a KS-test-based technique, we did not find a statistically significant disagreement between predicted and observed RGB star counts, over a brightness range of about 5 mag. The only problem concerns the brightness of the RGB bump, which appears to be fainter than theoretical expectations (by $\sim 0.15 \mathrm{mag}$ in $\left.K_{\mathrm{S}}\right)$ when adopting as reference values $(m-M)_{0}=13.18$, $[\mathrm{Fe} / \mathrm{H}]=-0.7, E(B-V)=0.04$ and an age of 11 Gyr. The discrepancy would disappear by allowing for an overshooting of $\sim 0.2 H_{p}$ beyond the bottom of the convective envelope in the theoretical stellar models. As an alternative, varying $[\mathrm{Fe} / \mathrm{H}]$, distance modulus and age within the associated errors, the discrepancy also vanishes, without the need to include any substantial amount of convective overshooting from the Schwarzschild boundary. The uncertainty on the spectroscopic metallicity and age prevent us from reaching a firm conclusion on this issue.

Furthermore, the star counts in the bump region (as parametrised by the quantity $R_{\text {bump }}^{K_{\mathrm{s}}}$ ) are not significantly different from theoretical expectations; this confirms the size of the H-profile discontinuity left over by the bottom boundary of the convective envelope at its largest extension, as predicted by theory.

Why is there no significant discrepancy in $K_{\mathrm{s}}$ (apart, possibly, from the bump level), whereas the LF in the optical as determined by Schiavon et al. (2002) discloses an inconsistency 
between theory and observations? At the moment it is not straightforward to find an answer to this question. As a test, we have considered the theoretical isochrones (from Salasnich et al. 2000; and Salaris \& Weiss 1998 including diffusion) employed by Schiavon et al. (2002), transformed to the 2MASS system using the same bolometric corrections adopted in this work. For both sets, the metallicity and $\alpha$-enhancement are the same as in the BaSTI models used here. The derived LFs for $11 \mathrm{Gyr}$ old RGB stars in $K_{\mathrm{s}}$ are identical to the theoretical LFs presented here, in the magnitude range relevant to our analysis. The only major difference is the brightness of the RGB bump, fainter in the isochrones of Salasnich et al. (2000) because of their inclusion of convective overshooting. A possible conclusion of this exercise is that the bolometric corrections to the $V$-band could be the reason for the discrepancy in the optical.

Acknowledgements. M.G. acknowledges support by MIUR, under the scientific project PRIN 2003029437. This publication makes use of data products from the Two Micron All Sky Survey, which is a joint project of the University of Massachusetts and the Infrared Processing and Analysis Center/California Institute of Technology, funded by the National Aeronautics and Space Administration and the National Science Foundation.

\section{References}

Alongi, M., Bertelli, G., Bressan, A., \& Chiosi, C. 1991, A\&A, 244, 95

Bonatto, C., Bica, E., \& Girardi, L. 2004, A\&A, 415, 571

Bono, G., Cassisi, S., Zoccali, M., \& Piotto, G. 2001, ApJ, 546, L109

Carretta, E., \& Gratton, R. G. 1997, A\&AS, 121, 95

Carretta, E., Gratton, R. G., Clementini, G., \& Fusi Pecci, F. 2000, ApJ, 533, 215

Carretta, E., Gratton, R. G., Bragaglia, A., Bonifacio, P., \& Pasquini, L. 2004, A\&A, 416, 925

Cassisi, S., degl'Innocenti, S., \& Salaris, M. 1997, MNRAS, 290, 515

Cassisi, S., Salaris, M., \& Bono, G. 2002, ApJ, 565, 1231

Cassisi, S., Salaris, M., Castelli, F., \& Pietrinferni, A. 2004, ApJ, 616, 498

Cho, D.-H., \& Lee, S.-G. 2002, AJ, 124, 977

Ferguson, J. W., Alexander, D. R., Allard, F., et al. 2005, ApJ, 623, 585

Ferraro, F. R., Montegriffo, P., Origlia, L., \& Fusi Pecci, F. 2000, AJ, 119, 1282
Fusi Pecci, F., Ferraro, F. R., Crocker, D. A., Rood, R. T., \& Buonanno, R. 1990, A\&A, 238, 95

Gibson, B. K., Madgwick, D. S., Jones, L. A., Da Costa, G. S., \& Norris, J. E. 1999, AJ, 118, 1268

Girardi, L., Bressan, A., Bertelli, G., \& Chiosi, C. 2000, A\&AS, 141, 371

Gratton, R. G., Bonifacio, P., Bragaglia, A., et al. 2001, A\&A, 369, 87

Grocholski, A. J., \& Sarajedini, A. 2002, AJ, 123, 1603

Gullieuszik, M., Held, E. V., Rizzi, L., et al. 2007, A\&A, 467, 1025

Jones, L. A., \& Worthey, G. 1995, ApJ, 446, L31

Kaluzny, J., Wysocka, A., Stanek, K. Z., \& Krzeminski, W. 1998, Acta Astron., 48, 439

Korn, A. J., Grundahl, F., Richard, O., et al. 2006, Nature, 442, 657

Kraft, R. P., \& Ivans, I. I. 2003, PASP, 115, 143

Liu, W. M., \& Chaboyer, B. 2000, ApJ, 544, 818

Momany, Y., Ortolani, S., Held, E. V., et al. 2003, A\&A, 402, 607

Ortolani, S., Renzini, A., Gilmozzi, R., et al. 1995, Nature, 377, 701

Percival, S. M., Salaris, M., van Wyk, F., \& Kilkenny, D. 2002, ApJ, 573, 174

Persson, S. E., Murphy, D. C., Krzeminski, W., Roth, M., \& Rieke, M. J. 1998, AJ, 116, 2475

Pietrinferni, A., Cassisi, S., Salaris, M., \& Castelli, F. 2004, ApJ, 612, 168 Pietrinferni, A., Cassisi, S., Salaris, M., \& Castelli, F. 2006, ApJ, 642, 797 Pulone, L. 1992, Mem. Soc. Astron. Ital., 63, 485

Reid, N. 1998, AJ, 115, 204

Reimers, D. 1975, Memoires of the Société Royale des Sciences de Liège, 8, 369

Renzini, A., \& Fusi Pecci, F. 1988, ARA\&A, 26, 199

Rieke, G. H., \& Lebofsky, M. J. 1985, ApJ, 288, 618

Rood, R. T. 1973, ApJ, 184, 815

Salaris, M., \& Girardi, L. 2002, MNRAS, 337, 332

Salaris, M., \& Weiss, A. 1998, A\&A, 335, 943

Salaris, M., Riello, M., Cassisi, S., \& Piotto, G. 2004a, A\&A, 420, 911

Salaris, M., Weiss, A., \& Percival, S. M. 2004b, A\&A, 414, 163

Salasnich, B., Girardi, L., Weiss, A., \& Chiosi, C. 2000, A\&A, 361, 1023

Salpeter, E. E. 1955, ApJ, 121, 161

Schiavon, R. P., Faber, S. M., Rose, J. A., \& Castilho, B. V. 2002, ApJ, 580, 873

Skrutskie, M. F., Cutri, R. M., Stiening, R., et al. 2006, AJ, 131, 1163

Stetson, P. B. 1994, PASP, 106, 250

Valenti, E., Ferraro, F. R., \& Origlia, L. 2004a, MNRAS, 354, 815

Valenti, E., Ferraro, F. R., Perina, S., \& Origlia, L. 2004b, A\&A, 419, 139

VandenBerg, D. A. 2000, ApJS, 129, 315

Vazdekis, A., Salaris, M., Arimoto, N., \& Rose, J. A. 2001, ApJ, 549, 274

Worthey, G. 1994, ApJS, 95, 107

Zinn, R., \& West, M. J. 1984, ApJS, 55, 45

Zoccali, M., Renzini, A., Ortolani, S., et al. 2001, ApJ, 553, 733 